\title{
Efficacy and safety of guidewireless catheterization with a steerable microcatheter in patients scheduled for yttrium-90 radioembolization: a prospective multicenter trial
}

\author{
Zbigniew Serafin ${ }^{1}$, Olivier Dudeck², Maciej Powerski ${ }^{3}$, Florian Wolf ${ }^{4}$, Ralph Drewes ${ }^{3}$, Maciej Pech ${ }^{3}$ \\ ${ }^{1}$ Department of Radiology and Diagnostic Imaging, Nicolaus Copernicus University, Collegium Medicum, Bydgoszcz, Poland \\ ${ }^{2}$ Center for Microtherapy, Klinik Hirslanden, Zurich, Switzerland \\ ${ }^{3}$ Department of Radiology and Nuclear Medicine, University of Magdeburg, Magdeburg, Germany \\ ${ }^{4}$ Division of Cardiovascular and Interventional Radiology, Medical University of Vienna, Vien, Austria
}

Videosurgery Miniinv 2020; 15 (3): 503-510

DOI: https://doi.org/10.5114/wiitm.2020.92512

\begin{abstract}
Introduction: A steerable, guidewireless microcatheter is of interest to interventional radiologists because it can improve effectiveness and reduce procedure timing and cost.

Aim: To assess the feasibility and safety of yttrium 90 radioembolization with a new steerable microcatheter without the need to use a guidewire.

Material and methods: A steerable microcatheter (Direxion; Boston Scientific, Natick, MA, USA) was prospectively used for yttrium-90 microsphere delivery in 50 patients (57 applications). The following factors were documented: vessel anatomy; shape and position of the guiding catheter; anticipated catheter position; technical success of catheterization; degree of difficulty in maneuvering; time to reach the target position; and complications.

Results: The microcatheter target position was in the right hepatic artery $(n=30,53 \%)$, the middle hepatic artery $(n=4,7 \%)$, the left hepatic artery $(n=19,33 \%)$, or in a segmental artery $(n=4,7 \%)$. Vessel catheterization was successful in 53 of 57 hepatic arteries (93\%). The mean time to reach the target position was $53.3 \pm 53.0 \mathrm{~s}$. The mean degree of difficulty in maneuvering the catheter to the target position was $2.3 \pm 1.3$ (easy) on a 6-point scale. The only complication observed was mild vasospasm in 1 patient.

Conclusions: The Direxion steerable microcatheter is characterized by unique steerability, allowing yttrium 90 delivery to be performed safely without use of a guidewire.
\end{abstract}

Key words: liver, radioembolization, steerable catheter.

\section{Introduction}

In recent years, the number of catheter-based endovascular procedures has gradually increased. In particular, novel transarterial locoregional anticancer treatments are increasingly being used, including gastrointestinal bleeding embolization, bland embolization, chemoembolization, and radioembolization (RE) [1].
A large variety of guidewire-directed microcatheters are commercially available today for such embolizations. The choice of microcatheter for catheterization of a particular target vessel depends on distal trackability, support, and pushability but also on the interventionalist's preference. In theory, all microcatheters can be shaped or reshaped with steam. In certain challenging endovascular procedures, steam shaping of the catheter tip may facilitate target ves-

\section{Address for correspondence}

Prof. Zbigniew Serafin, Department of Radiology and Diagnostic Imaging, Nicolaus Copernicus University, Collegium Medicum,

9 M. Skłodowskiej-Curie St, 85-094 Bydgoszcz, Poland, phone: +48 571377 599, e-mail: serafin@cm.umk.pl 
sel catheterization or provide the support needed to achieve a stable tip position for embolization [2]. However, steam shaping is time-consuming and therefore rarely done in clinical practice. Moreover, the steamed catheter end often recovers its initial shape [3].

A number of microcatheters with preshaped tips are available that improve selective catheterization of arteries originating at acute angles and help maintaining position within an aneurysm during coil embolization $[4,5]$. The range of shapes includes $45^{\circ}$ or $90^{\circ}$ angled tips, and J-, S- or C-shaped curves. The choice of a particular shape is related to the local anatomy and the intended method of treatment. However, these preshaped microcatheters are devoted mainly to neuroradiological interventions, which are performed in tortuous arteries and require a firm support during the treatment. On the other hand, oncological interventions in the visceral arteries require less support but more flexibility and steerability. The Direxion (Boston Scientific Corp., Natick, USA) is a new steerable microcatheter dedicated to visceral radiological interventions. It comes with various tip shapes and its design includes a proprietary Nitinol hypotube for steerability. Theoretically, guidewireless technique may reduce the time and cost of the procedure. In a recent comparative preclinical study on a porcine model, vessel catheterization without a guidewire was significantly more successful with the Direxion than with the Renegade (Boston Scientific, Natick, USA) steam-shaped catheter [6].

\section{Aim}

We conducted a clinical trial to verify the safety and efficacy of this new guidewireless catheterization technique in 50 consecutive patients scheduled for routine clinical yttrium-90 RE.

\section{Material and methods}

Fifty consecutive patients (32 male, 18 female; age $63.7 \pm 10.7$ years) scheduled for RE were prospectively enrolled in this multicenter clinical trial including three centers and three operators. The study was approved by the institutional review boards, and written informed consent was obtained from each patient. All patients underwent successful angiography with selective visceral catheterization, protective embolization of extrahepatic vessels originating from liver arteries to avoid migration of microspheres to the gastrointestinal tract, and treatment simulation with Tc-99m macroaggregated albumin (MAA). For hepatic yttrium-90 delivery, the femoral artery was cannulated under local anesthesia followed by placement of a 5 Fr sheath (Introducer II; Terumo Europe, Leuven, Belgium). The celiac trunk was catheterized, depending on the degree of its outlet angle, using a 5F Cobra (Imager II C2; Boston Scientific, Natick, MA, USA), a Soft-VU (Sos Omni Selective; Angiodynamics, Queensbury, NY, USA), or a Sidewinder II (Cordis, Miami, FL, USA) catheter. Liver target vessels were catheterized coaxially with a 0.021" Direxion (Boston Scientific Corp., Natick, USA) with a preshaped J-tip. This was attempted using guidewireless technique just by utilizing the ability to steer the tip of the microcatheter. The microcatheter was advanced during injection of contrast medium to optimize fluoroscopic guidance and to prevent trauma to target vessels. In cases when the optimal microcatheter position could not be reached using guidewireless technique, a microguidewire was used.

The interventional procedure was documented recording the following information: anatomy of the liver vessels according to the classification of Michels [7], type and anatomical position of the guiding catheter, anticipated catheter position for yttrium-90 delivery, success of the catheterization technique, degree of difficulty in maneuvering the microcatheter to the target position, time to reach the target position, and any complications. Each procedure was described by the interventionalist using a standardized questionnaire (Appendix 1) designed by our team.

\section{Statistical analysis}

Was performed with MedCalc v.18.10.2 (MedCalc Software bvba, Netherlands). A p-value of 0.05 was set to indicate statistical significance.

\section{Results}

The procedural features of the $57 \mathrm{RE}$ treatments in the 50 study patients are summarized in Table I. The vast majority of patients (82\%) had standard vascular anatomy (Michels I). Cobra II and Soft VU were the most commonly chosen guiding catheters, and they were typically placed in the celiac trunk.

The target position was reached in 53 RE treatments (93\%), and the mean time to reach the target position was $53.3 \pm 53.0$ s (range: 9-267 s). The reasons for unsuccessful catheterization with Direxion in four procedures were extremely tortuous vessel anat- 
Table I. Procedural features of 57 interventions in 50 study patients

\begin{tabular}{|c|c|c|c|c|}
\hline Liver anatomy & $\begin{array}{c}\text { Michels I: } \\
n=47 ; 82 \%\end{array}$ & $\begin{array}{l}\text { Michels II: } \\
n=4 ; 7 \%\end{array}$ & $\begin{array}{l}\text { Michels III: } \\
n=3 ; 5 \%\end{array}$ & $\begin{array}{c}\text { Michels > III: } \\
n=3 ; 5 \%\end{array}$ \\
\hline Y90 delivered via & $\begin{array}{c}\text { RHA: } \\
n=30 ; 53 \%\end{array}$ & $\begin{array}{c}\text { MHA: } \\
n=4 ; 7 \%\end{array}$ & $\begin{array}{c}\text { LHA: } \\
n=19 ; 33 \%\end{array}$ & $\begin{array}{c}\text { SA: } \\
n=4 ; 7 \%\end{array}$ \\
\hline Catheter used & $\begin{array}{c}\text { Cobra II: } \\
n=29 ; 51 \%\end{array}$ & $\begin{array}{c}\text { Soft VU: } \\
n=23 ; 40 \%\end{array}$ & $\begin{array}{c}\text { Sidewinder II: } \\
n=5 ; 9 \%\end{array}$ & - \\
\hline Position of guiding catheter & $\begin{array}{c}\text { CT: } \\
n=31 ; 54 \%\end{array}$ & $\begin{array}{c}\text { CHA: } \\
n=20 ; 35 \%\end{array}$ & $\begin{array}{c}\text { SMA: } \\
n=5 ; 9 \%\end{array}$ & $\begin{array}{c}\text { LGA: } \\
n=1 ; 2 \%\end{array}$ \\
\hline
\end{tabular}

RHA - right hepatic artery, MHA - middle hepatic artery, LHA - left hepatic artery, SA - segmental artery, CT - celiac trunk, CHA - common hepatic artery, $S M A$ - superior mesenteric artery, LGA - left gastric artery.
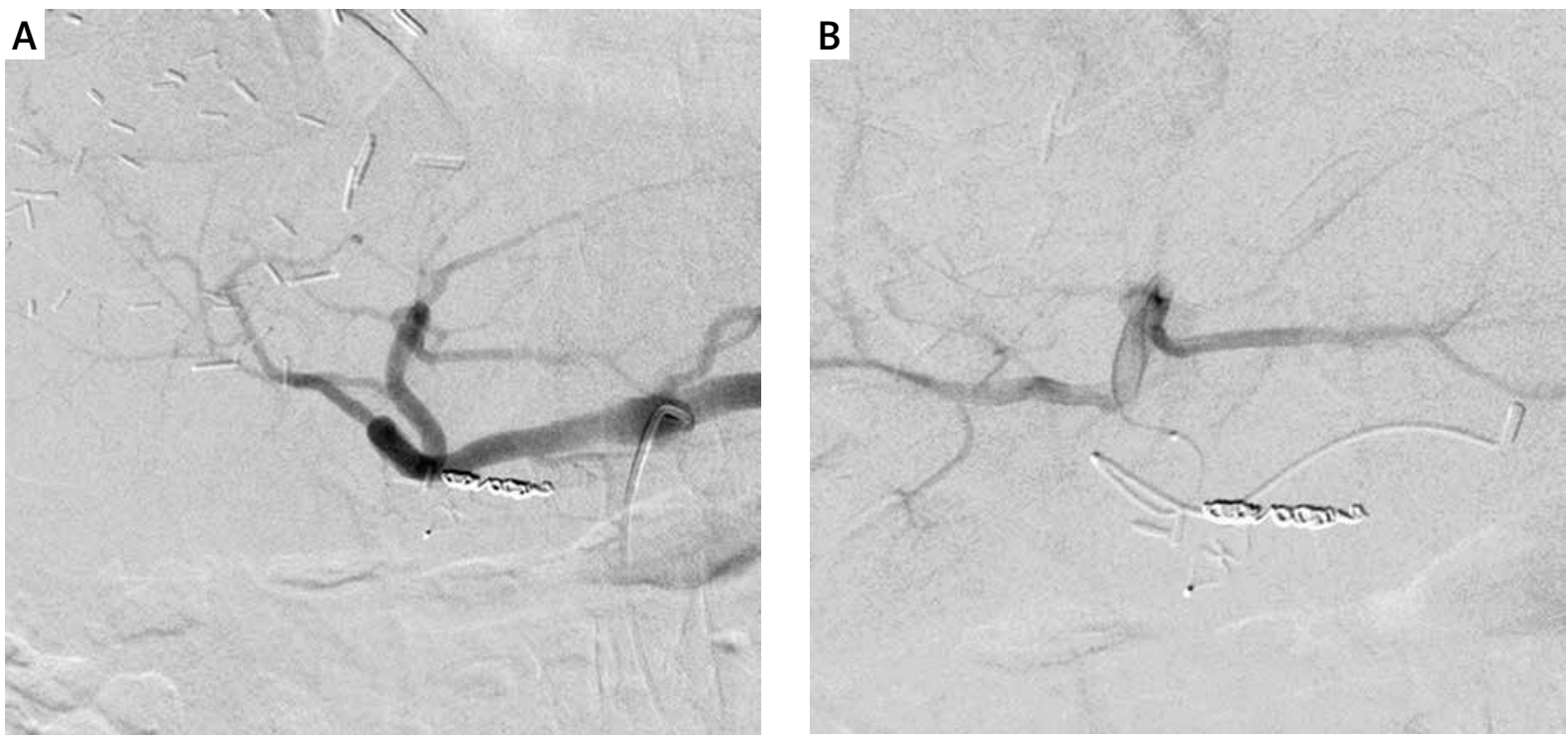

Photo 1. Example of unsuccessful catheterization with Direxion due to artery tortuosity. The target position could not be reached because of a very sharp angle between the left hepatic artery and its branch. Angiography from a guiding catheter $(\mathbf{A})$ and from a microcatheter (B)

omy $(n=2$; Photo 1$)$, very large parent artery diameter $(7.1 \mathrm{~mm}, n=1)$, which impeded precise steering because of the lack of support from the vessel wall (Photo 2), and celiac trunk occlusion ( $n=1$; Photo 3 ).

Yttrium-90 microspheres were delivered into the right hepatic artery in 53\% of cases, the left hepatic artery in 33\%, the middle hepatic artery in $7 \%$, and a segmental artery in 7\%. The technical difficulty to reach the target position was rated as easy with a mean score of $2.3 \pm 1.3$ (easy). The only complication associated with use of the Direxion catheter was a mild vasospasm in one instance.

The failure rate was too small for a statistical analysis of predisposing factors. In Spearman's rank correlation test, successful outcome was related only to the subjective difficulty score, which is an obvious conclusion. In the Kruskal-Wallis test, difficulty score, guiding catheter selection, and target vessel were statistically related to catheterization time ( $p<0.0001, p<0.001$, and $p<0.01$, respectively; Figures 1 and 2). Multiple regression analysis showed that guiding catheter selection and difficulty score were independent predictors of microcatheterization time. On the other hand, in receiver-operating characteristic (ROC) curve analysis, only target vessel was a significant predictor of the catheterization time but the area under the curve (AUC) value was only of borderline significance $(A U C=0.639,95 \% \mathrm{Cl}$ : 0.501-0.762).

\section{Discussion}

This study was the first to clinically document that the use of the Direxion microcatheter for guide- 


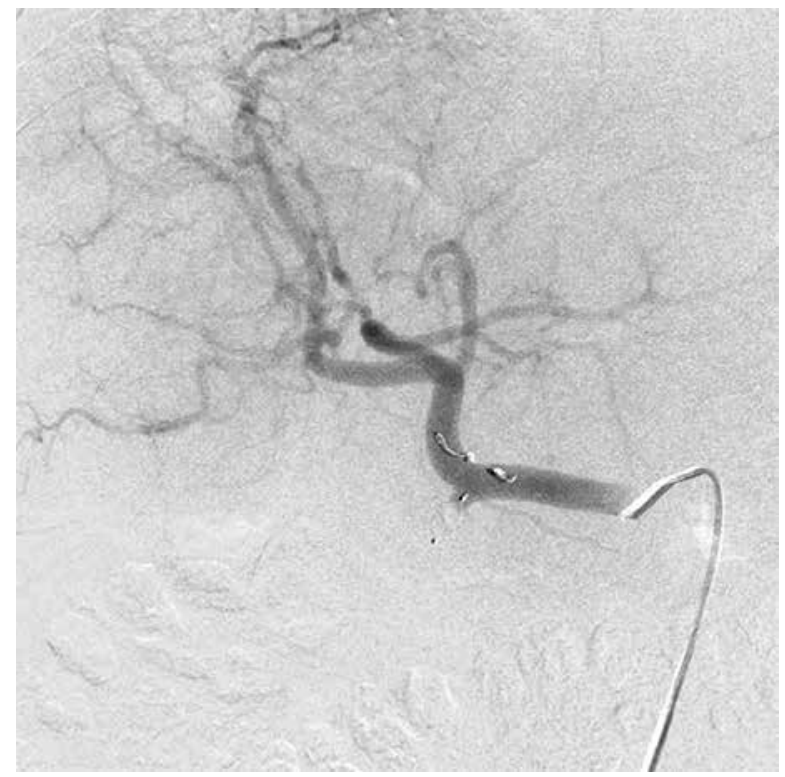

Photo 2. Example of unsuccessful catheterization with Direxion resulting from very wide arteries: diameter was $7.1 \mathrm{~mm}$ with a target vessel diameter of $5.8 \mathrm{~mm}$

wireless radioembolization is safe and has a very high technical success rate.

Liver-directed therapies for unresectable hepatic cancer are still evolving $[8,9]$. These treatments involve complex endovascular procedures that gener-

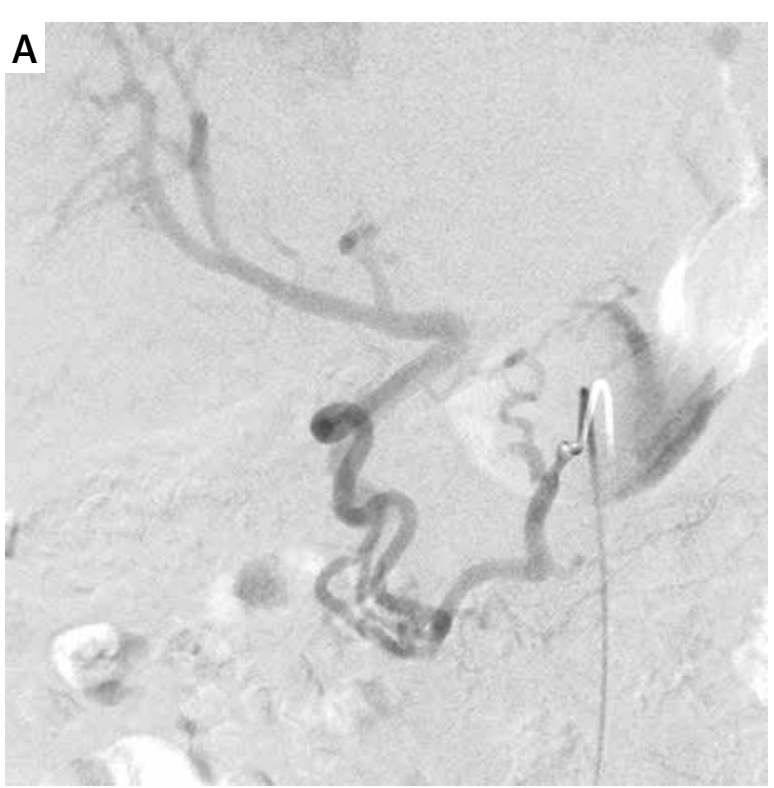

ally require the use of microcatheters. Particularly in RE, successful catheterization with prophylactic embolization of small and angled vessels, such as the right gastric artery, is crucial to prevent subsequent radiation-induced gastritis [10]. In contrast, therapeutic administration of yttrium-90 microspheres through the liver arteries is technically a rather simple interventional procedure. However, it still requires the use of a microcatheter for controlled delivery of the radionuclide.

Guidewireless catheterization is still uncommon in clinical practice, as it is associated with a significant risk of vessel injury and also of kinking of the catheter in its unsupported portions. Therefore, wireless microcatheterization has been considered an off-label procedure so far. However, in a preclinical study on a porcine model Dudeck found that catheterization without a guidewire with the Direxion was significantly more successful (88\%) compared with the Renegade (32\%) and also significantly faster than guidewire-assisted vessel catheterization with the Renegade (16.1 vs. $27.1 \mathrm{~s}$ on average) [6]. Another wireless microcatheter is SwiftNINJA (Merit Medical, South Jordan, UT, USA), which has so far been successfully used in single cases of kidney angiomyolipoma embolization with ethanol and iodized oil, in arterial infusion chemotherapy with coil

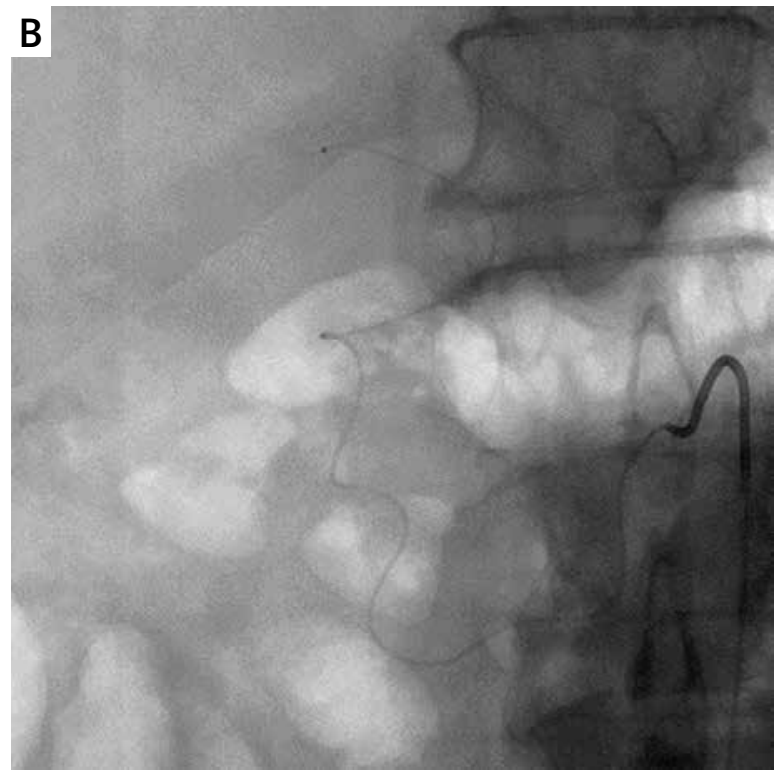

Photo 3. Example of unsuccessful catheterization with Direxion because of celiac trunk occlusion. Hepatic arteriography with the guiding catheter tip in the superior mesenteric artery (A). Direxion microcatheter in the proper hepatic artery (B). Note the very long and tortuous distance travelled by the microcatheter without support 


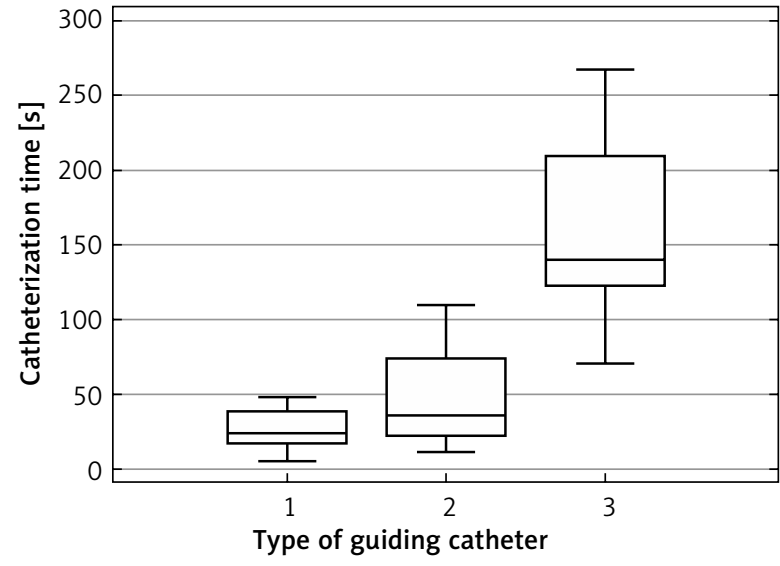

Figure 1. Boxplots showing relationship between guiding catheter and target catheterization time. 1 - Cobra, 2 - Sos-Omni, 3 - Sidewinder

embolization of maxillary cancer [11], and in a small multicenter trial of various types of procedures [12]. A unique feature of SwiftNINJA is its direct ergonomic steering dial that allows the catheter tip to be deflected from straight to $180^{\circ}$ in opposing directions. However, this device requires further clinical investigation before wider use. The third steerable microcatheter which is available on the market is Enzo ( $M i-$ crus Endovascular Corp, San Jose, California, USA). It was designed for intracranial applications and has a tip that can be deflected up to $90^{\circ}$ in opposing directions. However, there is only one published case report on the use of this microcatheter [13].

There appears to be a wide range of potential applications for steerable microcatheters in interventional radiology. Since Direxion microcatheters are currently only available for 0.021 " and 0.027 " systems, their application in neurovascular procedures may be limited to the delivery of stents and liquid embolics. Peripheral applications of the Direxion microcatheter seem to be much wider as it clearly enhances vessel selectivity in challenging anatomies where vessels cannot be accessed with standard systems [6]. Another potential advantage of Direxion is better control of coil loops during embolization through more precise microcatheter tip manipulations. Thus, positioning and packing of coils may be improved, reducing the risk of coil migration. Moreover, in the case of catheter kick-out during coil placement, repositioning of the microcatheter without a guidewire should be easier. The Direxion technology is also very promising for peripheral liquid embolization with glue or Onyx. A common

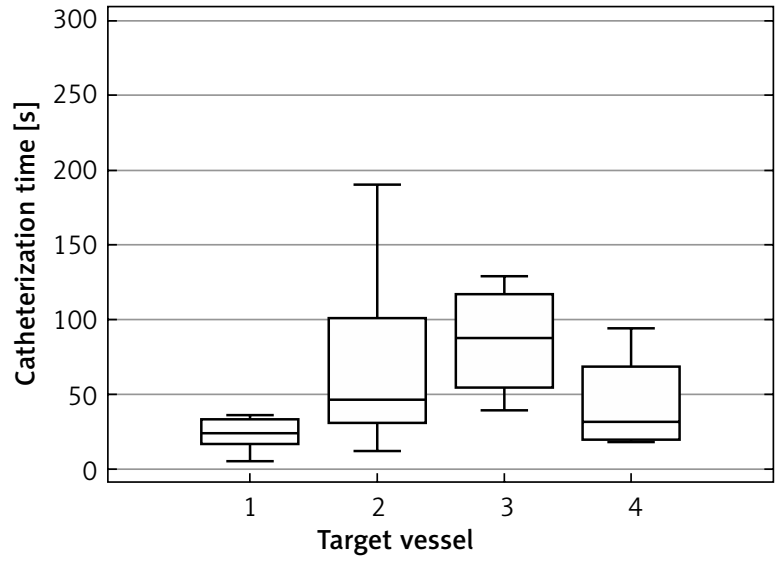

Figure 2. Boxplots showing relationship between target vessel and target catheterization time (s). 1 - right hepatic artery, 2 - left hepatic artery, 3 - middle hepatic artery, 4 - segmental hepatic artery

problem in such procedures is that significant repositioning of a microcatheter filled with an embolic agent is almost impossible since a guidewire cannot be inserted at this stage. In such situations, the use of a steerable microcatheter would be invaluable. The potential to reposition the microcatheter wirelessly could also be useful in patients undergoing RE, where radionuclide during workup angiography and the radioembolic agent for treatment need to be administered from different catheter positions. In such cases, the radiologist may be able to control the application and thus eliminate the risk of radioactive contamination that may otherwise result from insertion and removal of the guidewire.

The study has some limitations. Firstly, we treated a small random sample of patients from a general population with unselected anatomy. It is obvious that anatomy is a crucial factor influencing the success rate of target vessel catheterization, as seen in two of the unsuccessful cases in our study. However, the distribution of anatomic variants in our study population (Table I) is quite close to that of the general population. Secondly, our study population was also unselected regarding target vessels. Further studies should investigate and compare outcome of RE with the Direxion microcatheter in "easy" (right and left hepatic artery) and "demanding" (segmental arteries and the right gastric artery) vascular territories. Finally, in our study, we assessed only the immediate technical success rate without follow-up to detect potential later complications of 
the wireless technique. Nevertheless, our experience indicates that potential arterial injury that may be a complication of the use of a wireless catheter tends to be an immediate complication, as was the vasospasm seen in one of our patients.

\section{Conclusions}

The Direxion microcatheter is a novel device with good torque response. In this prospective clinical trial, Direxion allowed yttrium-90 delivery to be performed safely without the need for a guidewire in most of the patients.

\section{Conflict of interest}

Oliver Dudeck has participated in Scientific Advisory Boards for Boston Scientific. The remaining authors declare no conflict of interest.

\section{References}

1. Kennedy A, Coldwell D, Sangro B, et al. Integrating radioembolization ((90)Y microspheres) into current treatment options for liver tumors: introduction to the international working group report. Am J Clin Oncol 2012; 35: 81-90.

2. Ernst R, Tomsick T. Use of a coil introducer to shape microcatheters: AJNR Am J Neuroradiol 1994; 15: 521-2.

3. Abe T, Hirohata M, Tanaka N, et al. Distal-tip shape-consistency testing of steam-shaped microcatheters suitable for cerebral aneurysm coil placement. AJNR Am J Neuroradiol 2004; 25: 1058-61.

4. Kwon BJ, Seo DH, Ha YS, Lee KC. Endovascular treatment of wide-necked cerebral aneurysms with an acute angle branch incorporated into the sac: novel methods of branch access in 8 aneurysms. Neurointervention 2012; 7: 93-101.

5. Kelly ME, Fiorella D. Traumatic ophthalmic artery pseudoaneurysm coiled with a steerable microcatheter. Can J Neurol Sci 2009; 36: 496-9.

6. Dudeck O. Safety and efficacy of target vessel catheterization with the new steerable microcatheter Direxion compared with a standard microcatheter: a prospective, preclinical trial. Cardiovasc Intervent Radiol 2014; 37: 1041-6.

7. Michels NA. The hepatic, cystic and retroduodenal arteries and their relations to the biliary ducts with samples of the entire celiacal blood supply. Ann Surg 1951; 133: 503-24.

8. Coldwell DM, Sewell PE. The expanding role of interventional radiology in the supportive care of the oncology patient: from diagnosis to therapy. Semin Oncol 2005; 32: 169-73.

9. Johnston FM, Mavros MN, Herman JM, Pawlik TM. Local therapies for hepatic metastases. J Natl Compr Canc Netw 2013; 11: 153-60.

10. Lam MGEH, Banerjee S, Louie JD, et al. Root cause analysis of gastroduodenal ulceration after yttrium-90 radioembolization. Cardiovasc Intervent Radiol 2013; 36: 1536-47.
11. Soyama T, Yoshida D, Sakuhara Y, et al. The steerable microcatheter: a new device for selective catheterisation. Cardiovasc Intervent Radiol 2017; 40: 947-52.

12. Inaba Y, Arai Y, Sone M, et al. Clinical trial for development of a steerable microcatheter. Minim Invasive Ther Allied Technol 2019; 28: 1-5.

13. Kelly M, Fiorella D. The utilization of the Enzo steerable microcatheter for navigation of difficult anatomy in the treatment of a traumatic ophthalmic artery pseudoaneurysm. J Neurointerv Surg 2009; 1: 93.

Received: 23.08.2019, accepted: 19.11.2019. 


\section{Appendix 1}

\section{DIREXION CRF Questionnaire:}

Patient initials:

Date of birth:

Date of intervention:

Catheter type: Direxion 0.021

Direxion HIFLO 0.027

Tip shape: straight $\rightarrow$ individually shaped Bern $J$ Swan

Type of guiding catheter: Guiding catheter position: celiac trunk, common hepatic artery

SIRT:

Radioembolization procedure: via right hepatic artery via left hepatic artery via middle hepatic artery via segmental artery other

Anticipated catheter position for SIRT was accessible with Direxion only: yes, no

If no, give reason(s):

Time needed to position Direxion: $\min$ sec

(start with Direxion at the tip of the guiding catheter until anticipated catheter position for SIRT is reached)

Rate difficulty to reach anticipated catheter position for SIRT

1 (very easy)

3 (possible, but difficult) 2 (easy)

5 (impossible without wire)

Complications: yes, no

If yes, specify:

If needed, microguidewire used: 


\section{COILING:}

Additional coiling needed to occlude hepatoenteric collaterals:

yes, no

Vessel size: $\mathrm{mm}$

Vessel access possible with Direxion alone: yes, no

If no, give reason(s):

Rate difficulty to reach target vessel:

1 (very easy)

3 (possible, but difficult)

5 (impossible without wire)
2 (easy)

4 (hardly possible)

6 (challenging even with wire)

Complications: yes, no

If yes, specify:

If needed, microguidewire used:

\section{CATHETERIZATION OF SECOND VESSEL:}

Time needed to reposition Direxion: $\min \quad \mathrm{sec}$

(start with Direxion at first SIRT position until second anticipated catheter position is reached)

Anticipated catheter position for SIRT was accessible with Direxion alone: yes, no

If no, give reason(s):

Rate difficulty to reach anticipated catheter position for SIRT
1 (very easy) 2 (easy)
3 (possible, but difficult) 4 (hardly possible)
5 (impossible without wire) 6 (challenging even with wire)

Complications: yes, no

If yes, specify:

If needed, microguidewire used: 